\title{
Zinc Oxide Nanoparticles Prepared By a Simple Heating: Effect of Polymer Addition and Polymer Absence on the Morphology
}

\author{
Mikrajuddin Abdullah $^{1,2}$ \& Kikuo Okuyama ${ }^{2}$ \\ ${ }^{1}$ Department of Physics, Bandung Institute of Technology \\ Jl. Ganeca 10 Bandung 40132, Indonesia \\ E-mail: din@hiroshima-u.ac.jp \\ ${ }^{2}$ Department of Chemical Engineering \\ Graduate School of Engineering, Hiroshima University \\ 1-4-1 Kagamiyama, Higashi Hiroshima 739-8527, Japan
}

\begin{abstract}
Zinc oxide ( $\mathrm{ZnO})$ nanoparticles were prepared by a simple heating of precursors in a furnace at temperatures of below $1000^{\circ} \mathrm{C}$ in an air environment. If zinc nitrate was used as precursor, polymer (e.g., polyethylene glycol (PEG)) must be added into the precursor to produce $\mathrm{ZnO}$ in nanometer size. The absence of polymer led to the presence of several micrometer-sized flakes. In addition, the heating temperatures must be higher than $500^{\circ} \mathrm{C}$ to completely decompose the organic material in final product. However, if zinc acetate was used as precursor, nanometer-sized $\mathrm{ZnO}$ having a high crystallinity can be obtained even when the polymer was absent. Interestingly, we also found that heating at low temperatures (e.g. $400^{\circ} \mathrm{C}$ ) resulted in $\mathrm{ZnO}$ nanorods with an elongation ratio of around 5. This method is rapid, economically efficient, and readily scalable for industrial applications.
\end{abstract}

Keyword: Zinc oxide nanoparticles; zinc oxide nanorods; crystallinity, particle size.

\section{$1 \quad$ Introduction}

$\mathrm{ZnO}$ is frequently used as a reinforcing filler (vulcanizing agents) for elastomers and is used in pharmaceuticals, cosmetics, raw materials for varistor, phosphor and ferrite, catalyst, sensors, etc. ${ }^{1}$ Recently, research on $\mathrm{ZnO}$ nanoparticles having diameter of several nanometers has attracted a lot of attentions. Nanoparticles and nanostructured materials comprised of metals and semiconductors that exhibit size dependent optical, magnetic, electronic and catalytic properties have recently been heralded as the next generation of electronic devices in the design of advanced materials. $\mathrm{ZnO}$ emits a broad luminescence emission in the green-yellow region, and, as a result, it is a potential material for use in white light sources. When the particle size is reduced below $6 \mathrm{~nm}$, a shift in the emission spectrum to shorter wavelengths due to the quantum confinement of electrons and holes in the particles occurs. 
The peak for the emission spectrum is located in the blue region for particle sizes smaller than about $3 \mathrm{~nm}$. Very large $\mathrm{ZnO}$ particles show an emission spectrum that peaks at a wavelength of around $550 \mathrm{~nm}$, i.e., a green-yellow color. We have reported the manipulation of $\mathrm{ZnO}$ nanoparticles properties to produce various luminescence colors. ${ }^{2-8}$

Although research on nanometer size particles (below $10 \mathrm{~nm}$ ) have attracted considerably attention, particles in the submicrometer size or several tens of nanometer in size continue to be important for current applications. For example, it is not necessary for phosphor particles for use in displays to have sizes down to several nanometers. The pixel in a display, which usually is several tens of micrometers in size can produce very high quality pictures, and this pixel can be well developed using submicrometer size particles. In this technology, the most significant point to be explored is a rapid and simple method for producing particles in the sub micron down to several tens of nanometer size that emit a high luminescence intensity. Another fact to be considered is that, the post-processing of submicrometer sizes particles is much easier than for particles that are several nanometer in size.

Motivated by previous studies of the preparation of oxide particles in submicron down to several tens of nanometer sizes, ${ }^{9-12}$ the objective of this paper is the production of a zinc oxide in a hundreds of nanometer size. The morphology of zinc oxide sample prepared with and without polymer will be compared. This method has potential for making zinc oxide nanoparticles in a large scale for use in industry.

\section{$2 \quad$ Experimental}

\subsection{Preparation of $\mathrm{ZnO}$ from Zinc Nitrate Precursor}

Zinc nitrate hexahydrate, $\mathrm{Zn}\left(\mathrm{NO}_{3}\right)_{2} \cdot 6 \mathrm{H}_{2} \mathrm{O}(>99.0 \%$, Kanto Chemicals, Japan), was dissolved in $10 \mathrm{~mL}$ of ultra pure water and stirred until a clear solution was obtained. Various weights of polyethylene glycol, $\mathrm{H}(\mathrm{EG})_{\mathrm{n}} \mathrm{H}$, with $\mathrm{n}=20000$ and $\mathrm{EG}=\mathrm{OCH}_{2} \mathrm{CH}_{2}$ (Wako Pure Chemicals, Japan): $0 \mathrm{~g}, 2 \mathrm{~g}, 4 \mathrm{~g}$, and $8 \mathrm{~g}$, were added to the solution, followed by heating under stirring at $70^{\circ} \mathrm{C}$ for $20 \mathrm{~min}$ to produce viscous precursor solution. The precursor was then heated in a furnace at various temperatures for $30 \mathrm{~min}$ in air.

\subsection{Preparation of $\mathrm{ZnO}$ from Zinc Acetate Precursor}

Zinc acetate dihydrate, $\mathrm{Zn}\left(\mathrm{CH}_{3} \mathrm{COO}\right)_{2} \cdot 2 \mathrm{H}_{2} \mathrm{O}(>99.0 \%$, Kanto Chemicals, Japan), was dissolved in $10 \mathrm{~mL}$ of ultra pure water and stirred until a clear 
solution was obtained. The precursor was then heated in a furnace at various temperatures for $30 \mathrm{~min}$ in air.

\subsection{Characterizations}

X-ray diffraction (XRD) patterns were obtained using a Rigaku Denki RINT2000 instrument ( $\mathrm{CuK} \alpha$ source, $\lambda=1.5418 \AA)$. Scanning electron micrograph (SEM) pictures were obtained using a field emission SEM (FESEM Hitachi S-5000) instrument at $20 \mathrm{kV}$.
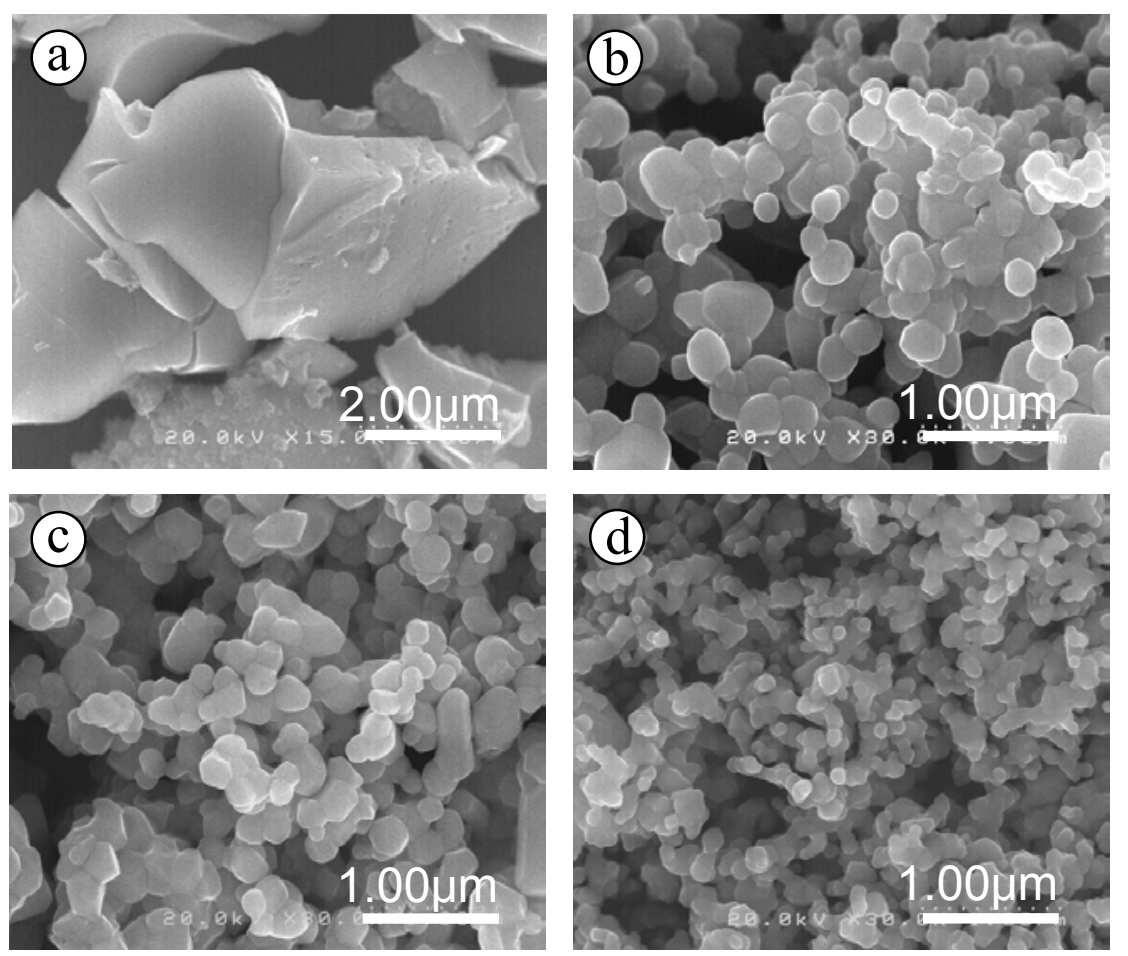

Figure 1 SEM pictures of samples, showing the effect of PEG content on the morphology: (a) no PEG, (b) PEG 4 g, (c) PEG 8 g, and (d) PEG 12 g. Various polymer weights were added in $10 \mathrm{~mL}$ solution of zinc nitrate $0.6 \mathrm{M}$. The precursors were heated at $800^{\circ} \mathrm{C}$ for $30 \mathrm{~min}$.

\section{$3 \quad$ Results and Discussion}

Figure 1 is SEM images, showing the effect of polymer fraction on the particle morphology in sample prepared using zinc oxide precursor $0.6 \mathrm{M}$. The precursors were heated at $800^{\circ} \mathrm{C}$ for $30 \mathrm{~min}$ in air atmosphere. When polymer was absent, large flakes of several nanometer sizes were found. Coagulation 
process intensively occurred during the heating process since the absence of separating medium between particles to permit the as produced particles coalescence easily. Adding polymer in the precursors dramatically reduced the particle size. The large flakes in sample prepared without polymer disappeared. Before decomposition, polymer played a role as separating medium to overcome coalescence of the as product particles so that coagulation can be avoided (reduced). Smaller particle sizes were obtained when using higher fraction of polymer. Adding more polymer, overcome extensively the coalescence between the as produced particles of that the final particle sizes decreased. Particle size of about 300, 200, and less than $100 \mathrm{~nm}$ were obtained when adding, respectively, $4 \mathrm{~g}, 8 \mathrm{~g}$, and $12 \mathrm{~g}$ polymers.

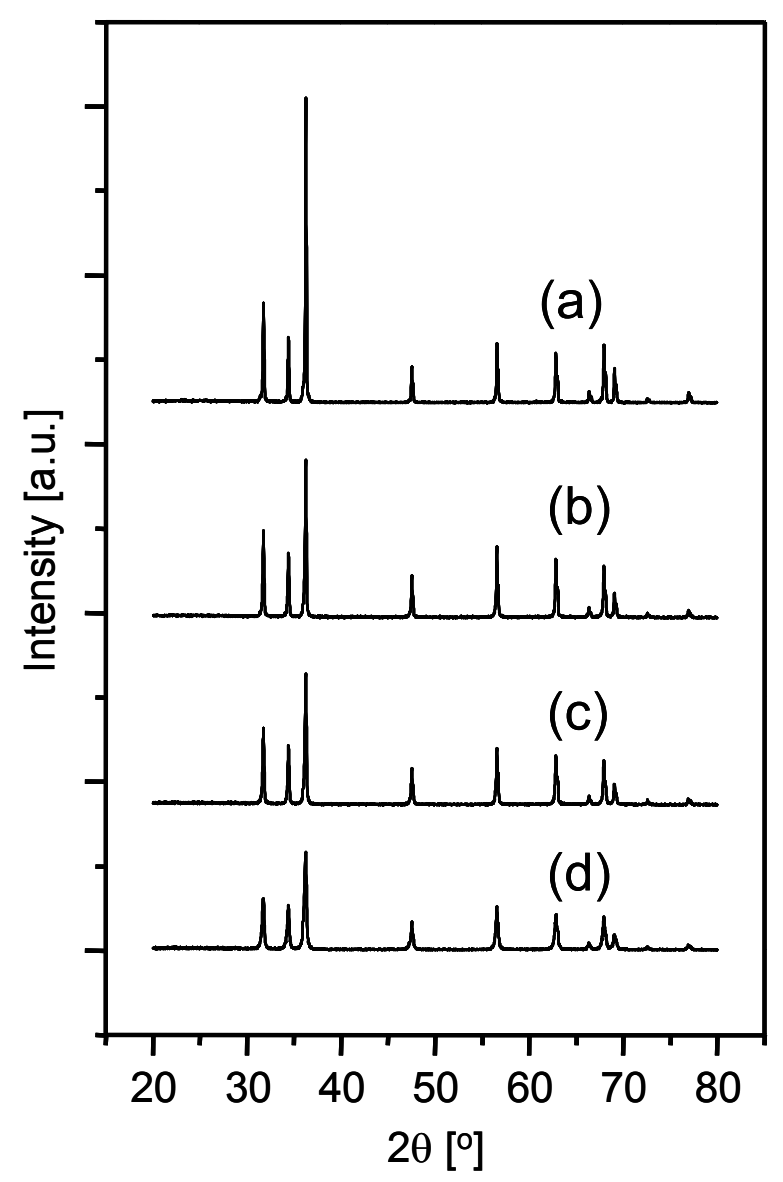

Figure 2 Powder XRD patterns of samples, showing the effect of PEG content on the crystallinity: (a) no PEG, (b) PEG 4 g, (c) PEG 8 g, and (d) PEG 12 g. Various polymer weights were added in $10 \mathrm{~mL}$ solution of zinc nitrate $0.6 \mathrm{M}$. The precursors were heated at $800^{\circ} \mathrm{C}$ for $30 \mathrm{~min}$. 
Figure 2 is the powder X-ray diffraction (XRD) patterns of sample prepared at various polymer fractions. The highest crystalline state was observed in sample prepared without polymer. For samples prepared with polymer, the crystallinity (the intensity of XRD peaks) decreased with increasing the polymer fraction added into the precursors.
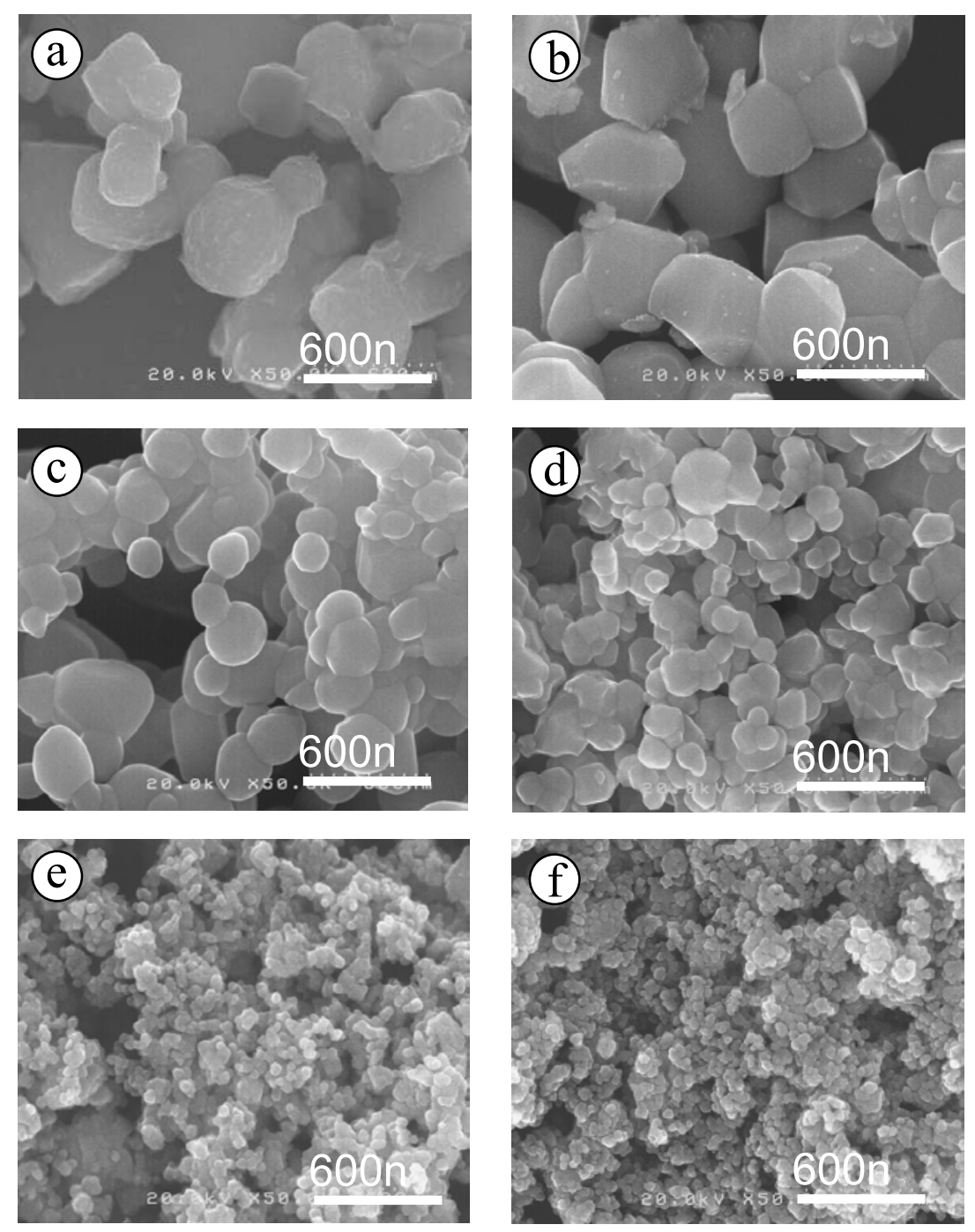

Figure 3 SEM pictures of samples, showing the effect of heating temperature on the morphology: (a) $1000^{\circ} \mathrm{C}$, (b) $900^{\circ} \mathrm{C}$, (c) $800^{\circ} \mathrm{C}$, (d) $700^{\circ} \mathrm{C}$, (e) $600^{\circ} \mathrm{C}$, and (f) $500^{\circ} \mathrm{C}$. PEG $8 \mathrm{~g}$ was added in $10 \mathrm{~mL}$ solution of zinc nitrate $0.6 \mathrm{M}$ and the precursors were heated for $30 \mathrm{~min}$. 
Various heating temperature were tried to investigate the effect of temperature on the sample morphology. SEM images in Fig. 3 are the morphology of sample processed at various temperatures. The processing time were fixed for $30 \mathrm{~min}$ and the amount of polymer used was fixed at $8 \mathrm{~g}$. The particle size increased with heating temperature. This observation will be briefly explained later. The corresponding XRD patterns are displayed in Fig. 4. When temperature increased, initially the intensity increased and then appreciable unchanged when temperatures are higher than $800^{\circ} \mathrm{C}$. It informs that $800^{\circ} \mathrm{C}$ is the optimum temperature since we obtained the highest crystallinity and appreciably small particle size. The heating temperatures above $800^{\circ} \mathrm{C}$ does not change the crystallinity, but changed the particle size significantly.

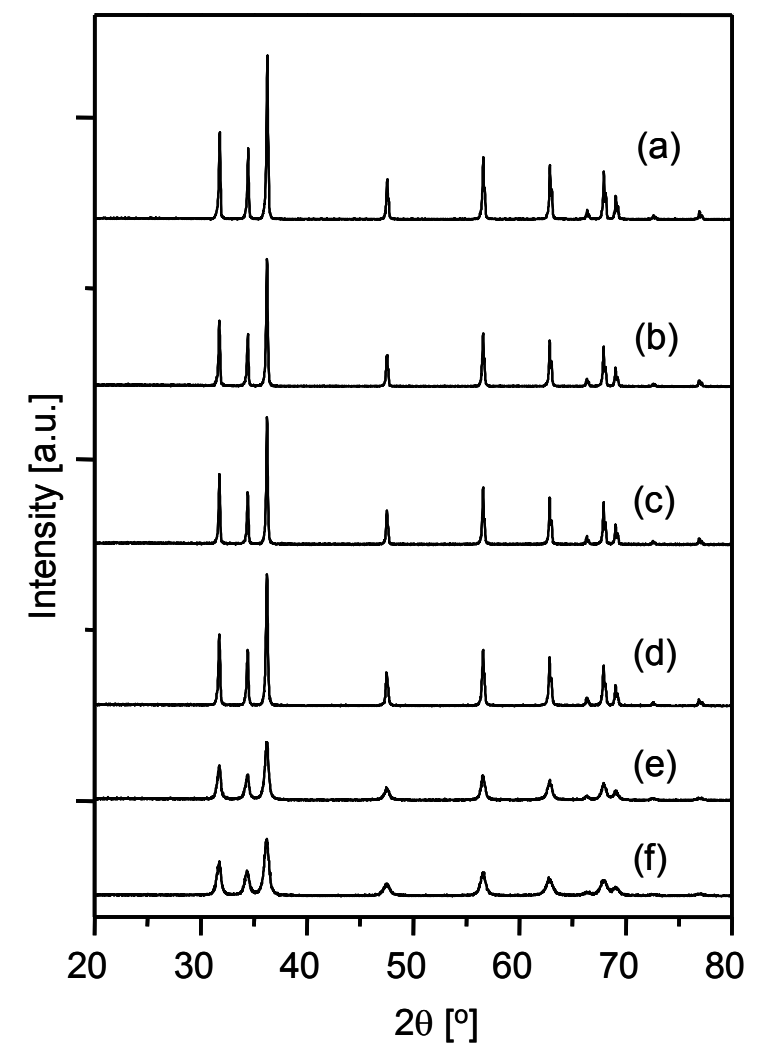

Figure 4 Powder XRD patterns of samples, showing the effect of heating temperature on the crystallinity: (a) $1000^{\circ} \mathrm{C}$, (b) $900^{\circ} \mathrm{C}$, (c) $800^{\circ} \mathrm{C}$, (d) $700^{\circ} \mathrm{C}$, (e) $600^{\circ} \mathrm{C}$, and (f) $500^{\circ} \mathrm{C}$. PEG $8 \mathrm{~g}$ was added in $10 \mathrm{~mL}$ solution of zinc nitrate $0.6 \mathrm{M}$ and the precursors were heated for $30 \mathrm{~min}$. 
We predicted the crystalline sizes in sample from XRD patterns using Scherrer formula

$$
D=\frac{0.9 \lambda}{B \cos \theta_{B}}
$$

with $D$ the crystalline diameter, $\lambda$ the wavelength of X-ray, and $\theta_{B}$ the Bragg angle. $B$ the line broadening obeys the Warren formula

$$
B^{2}=B_{M}^{2}-B_{S}^{2},
$$

with $B_{M}$ the measured peak width at half of the peak height of the sample and $B_{S}$ the corresponding width of a standard material having large crystalline size mixed with the sample with diffraction peak near the relevant peak of the sample. ${ }^{13}$ For large crystallines, the XRD peaks are very narrow, while for nanocrystallines, the XRD peaks are broad. Thus, for simplicity, we assume $B_{M}$ $>B_{S} \quad$ so that $B \cong B$. Figure 5 shows the effect of heating temperature on the crystalline sizes. Crystalline sizes down to $20 \mathrm{~nm}$ was obtained for sample prepared at $500^{\circ} \mathrm{C}$, and then rapidly increased with temperature up to $800^{\circ} \mathrm{C}$ at which the crystalline size was about $60 \mathrm{~nm}$. Above $800^{\circ} \mathrm{C}$, the crystalline size did not change so much from $60 \mathrm{~nm}$.

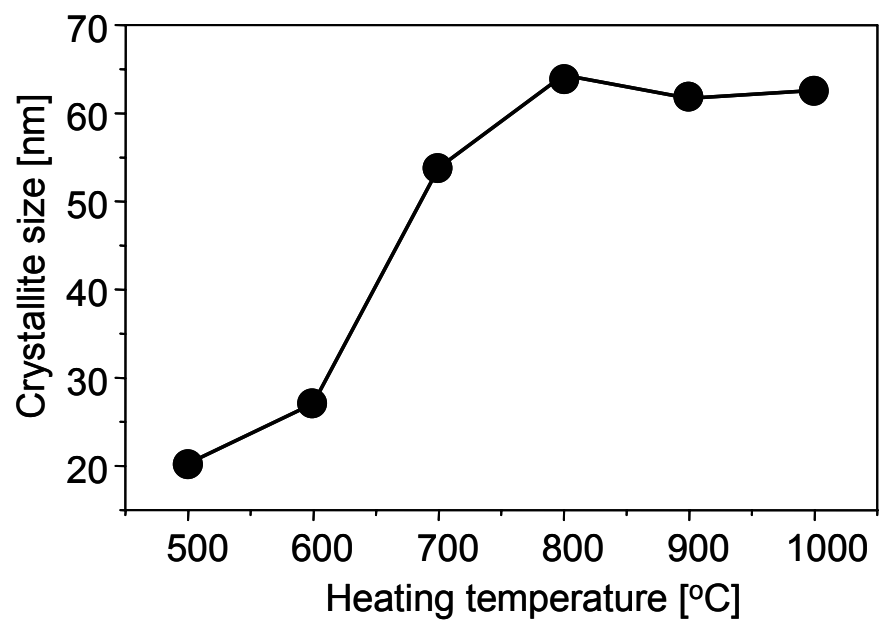

Figure 5 Effect of heating temperature of the crystalline size of samples (predicted using Warren-Scherrer formula based on the XRD patterns). The preparation temperatures are: (a) $1000^{\circ} \mathrm{C}$, (b) $900^{\circ} \mathrm{C}$, (c) $800^{\circ} \mathrm{C}$, (d) $700^{\circ} \mathrm{C}$, (e) $600^{\circ} \mathrm{C}$, and (f) $500^{\circ} \mathrm{C}$. PEG $8 \mathrm{~g}$ was added in $10 \mathrm{~mL}$ solution of zinc nitrate $0.6 \mathrm{M}$ and the precursors were heated for $30 \mathrm{~min}$. 
If we compare the predicted crystalline size with the particle size observed by SEM in Fig. 3, we found that the particle size observed by SEM is larger that the crystalline size. It suggests that particles in Fig. 3 are not single crystals. These particles are composed of nanocrystallites.

Here we suggest a simple mechanism that control the entire particle formation. During heating process, mainly there are two mechanism took place. At the initial stage of heating, primary particles, the size of which is near to that of crystallites, are formed. The size of those primary particles is mainly controlled by a chemical reaction. The chemical reaction rate can be simply written as $d C /$ $d t=-k C^{2}$, with $C$ is the precursor concentration and $k$ is the reaction constant, satisfies an Arrhenius law, $k \propto \exp \left[-E_{a} / k_{B} T\right]$. Here $E_{a}, k_{B}$, and $T$ are the activation energy, the Boltzmann constant, and the temperature, respectively. It is clear that $k$ increases with temperature, which in turn, resulting in the reaction rate and primary particle size (crystallines size) increase with temperature, which in agreement with Fig. 5.

The second process is coagulation of primary particles. The rate of growth of cluster containing $k$-primary particles (we call $k$-mer) can be written as ${ }^{14}$

$$
d N_{k}(t) / d t=(1 / 2) \sum_{j=1}^{k-1} K_{j, k-j} N_{j} N_{k}-N_{k} \sum_{j=1}^{\infty} K_{k, j} N_{j},
$$

where $N_{k}$ is the concentration of particles containing $k$-mer and $K_{j, k}$ is the coagulation coefficient between particles containing $j$ and $k$-mers. $K_{j, k}$ can be calculated from the Fuchs equation ${ }^{15}$ or the Dahneke equation. ${ }^{16}$ If the medium around the primary particles is, either an aqueous solution or a polymer melt, we can assume that the primary particles are located in continuum region. To understand the role of temperature on particle size, let us roughly assume that $K_{j, K}=K$. Solving the discrete coagulation equation leads to the concentration of particles at time $t$ at a relatively long time compared to the characteristic time $\tau_{c}=2 / K N_{0}$, is given by

$$
N(t)=2 / K t,
$$

where $N_{0}$ is the number concentration of particles at $\mathrm{t}=0$. Assuming that the primary particles are similar in size, one can approximate $\mathrm{K}=8 \mathrm{kT} / 3 \mu$, with $k$ the Boltzmann constant, $T$ the absolute temperature and $\mu$ the viscosity. ${ }^{14}$ Although this relation was valid for the agglomeration of colloid particles under the condition of ignorable interparticle potential, ${ }^{17}$ we assume that it can also be used to explain the agglomeration process in a polymer liquid by replacing the viscosity of liquid in the colloid with the viscosity of the polymer liquid when calculating the coagulation coefficient. It is clear that the particle number 
concentration increases with increasing viscosity, and therefore, the particle size decreases with increasing viscosity.

There are serious problems faced by preparation of particles with polymer. First is the use of polymer itself, which will be burnt during the process, so that economically this process is inefficient. In addition, since the used of organic material that burnt during process, there is a potential for the presence of residual carbon material in the sample. Finally, since organic material must be removed to obtain pure oxide, precursor must be heated at high temperatures to decompose organic material so that low temperature processing is not allowed in this process. We observed, when using a PEG as polymer, the heating temperature must be higher than $500^{\circ} \mathrm{C}$. Heating at temperatures of less that 500 ${ }^{\circ} \mathrm{C}$ leaving a large amount of carbon residue and the sample color was black.
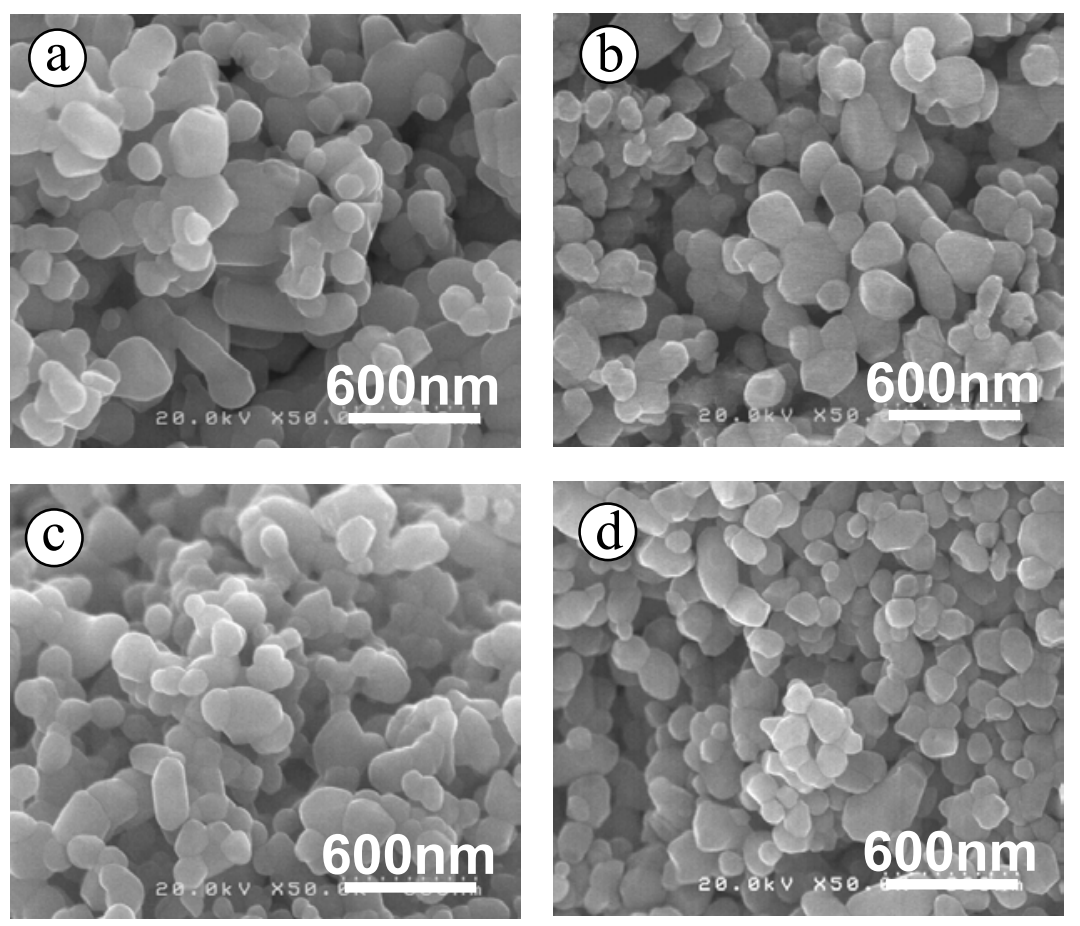

Figure 6 SEM pictures of samples, showing the effect of PEG content on the morphology: (a) no PEG, (b) PEG 2 g, (c) PEG 4 g, and (d) PEG 8 g. Various polymer weights were added in $10 \mathrm{~mL}$ solution of zinc acetate $0.6 \mathrm{M}$. The precursors were heated at $800^{\circ} \mathrm{C}$ for $30 \mathrm{~min}$.

Thus, it is challenging to seek another route so that nanoparticles can be produced without polymer. In addition, since polymer is absent, low 
temperature processing is also permissible. We found, the use of zinc acetate as precursor, instead of zinc nitrate, is permissible to achieve this goal.

Figure 6 is the SEM pictures of sample prepared using zinc acetate $0.6 \mathrm{M}$, heated at $800^{\circ} \mathrm{C}$ for $30 \mathrm{~min}$. Various fractions of PEG were added into the precursors. We see that the sample morphology is independent of polymer fraction. The particle size is almost similar in samples, weather prepared with polymer or prepared with polymer at various polymer contents. It suggests that, when using zinc acetate as the precursor, we don't need to add polymer to find zinc oxide nanoparticles. The XRD patterns of sample in Fig 6 are shown in Fig. 7. Interestingly, the crystallinity of all sample are very similar independent of polymer content in the precursor.

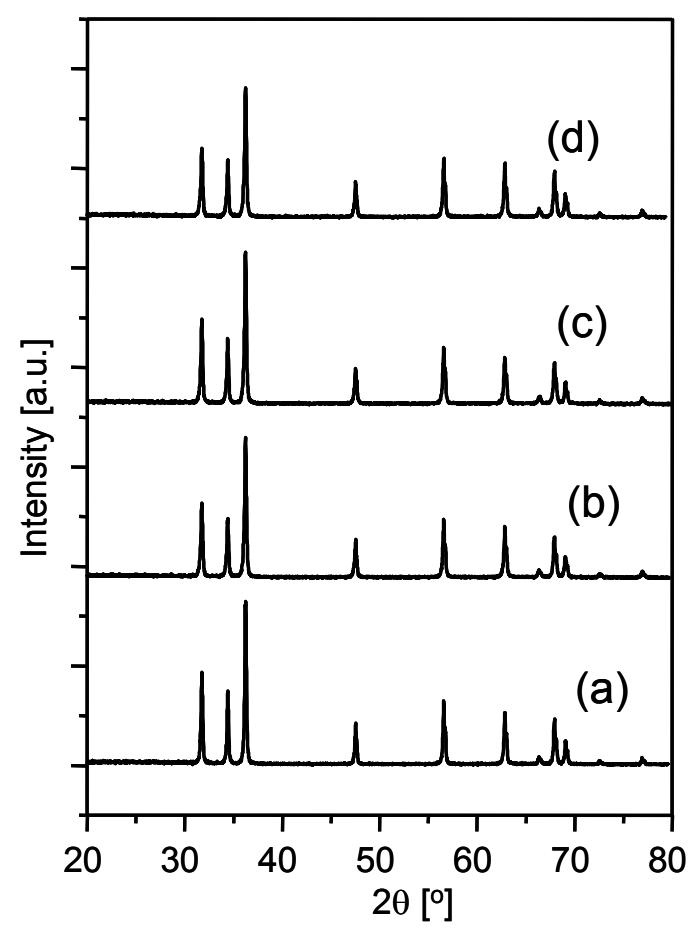

Figure 7 Powder XRD patterns of samples, showing the effect of PEG content on the crystallinity: (a) no PEG, (b) PEG 2 g, (c) PEG 4 g, and (d) PEG $8 \mathrm{~g}$. Various polymer weights were added in $10 \mathrm{~mL}$ solution of zinc acetate 0.6 M. The precursors were heated at $800^{\circ} \mathrm{C}$ for $30 \mathrm{~min}$.

Figure 8 shows the effect of temperature on the sample morphology. No polymer was added in the precursor. The processing time was $30 \mathrm{~min}$. The particle size also increased with temperature, similar to that observed in sample prepared with zinc nitrate. Interestingly, at $400^{\circ} \mathrm{C}$ we observed rod-like particles 
with elongation ratio (length/diameter) of around 5. This particle shape can not be obtained when using zinc nitrate precursor since using this precursor, the heating temperature can not be set down to $400^{\circ} \mathrm{C}$. This observation suggests that the present method combined with the use of zinc acetate precursor is a simple route to produce $\mathrm{ZnO}$ nanorods.
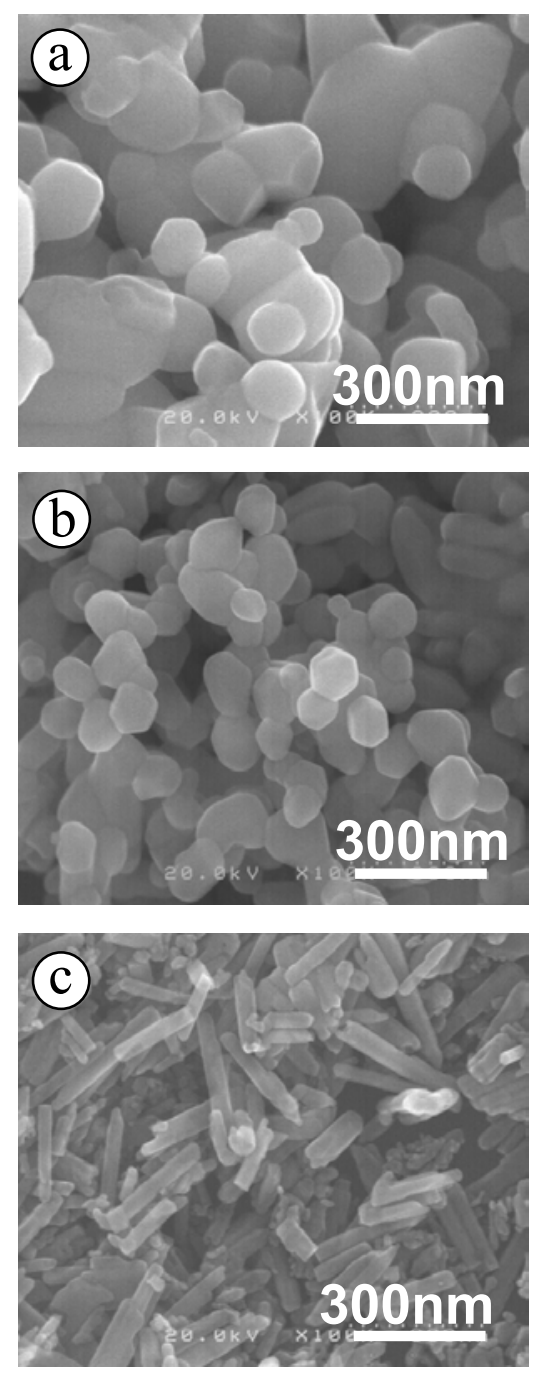

Figure 8 SEM pictures of samples, showing the effect of heating temperature on the morphology: (a) $800^{\circ} \mathrm{C}$, (b) $600^{\circ} \mathrm{C}$, and (c) $400^{\circ} \mathrm{C}$. The precursors are 10 $\mathrm{mL}$ solutions of zinc acetate $0.6 \mathrm{M}$, heated for $30 \mathrm{~min}$. 
The most important of the present method is it is rapid, economic, and scalable. Continuous processing is also ready to be developed since the entire process is very simple, i.e., just a simple heating. Heater with operation temperature of around $800^{\circ} \mathrm{C}$ can be designed easily. Finally, although we reported here the production of zinc oxide, indeed, this method is also applicable for production of various oxide materials in the form of nanoscale powders for a huge number of applications.

\section{Conclusion}

Nanometer-sized $\mathrm{ZnO}$ can be produced by a simple heating of precursor solution containing zinc nitrate and PEG. The particles sizes are less that $300 \mathrm{~nm}$ and crystalline size are less than $60 \mathrm{~nm}$ when heating temperatures were below $1000^{\circ} \mathrm{C}$. Both particle size and crystallinity increase with heating temperature. The particle size decreases with increasing polymer content. However, adding a polymer in the precursor does not permit a low temperature heating (less than $500^{\circ} \mathrm{C}$ ) since will result in the presence of organic/carbon residue in the final product. An alternative and potential route is by using a solution of zinc acetate as precursor. Even when polymer is absent, nanometer-sized $\mathrm{ZnO}$ can still be obtained. This method also permits a low temperature heating of the precursor. Heating at $400^{\circ} \mathrm{C}$ results in $\mathrm{ZnO}$ nanorods with an elongation ratio of around 5 . This method is rapid, economically efficient, and readily scalable for industrial applications.

\section{References}

1. Tani, T., Madler, L. \& Prastinis, S. E., Homogeneous $\mathrm{ZnO}$ nanoparticles by flame spray pyrolysis, J. Nanoprt. Res. 4, pp. 337-343 (2002).

2. Mikrajuddin, Iskandar, F., Shi, F. G. \& Okuyama, K., Stable photoluminescence of zinc oxide quantum dots in silica nanoparticles matrix, J. Appl. Phys. 89, pp. 6431-6434 (2001).

3. Mikrajuddin, Lenggoro, I. W., Shi, F. G. \& Okuyama, K., Luminescent polymer electrolytes nanocomposoites prepared by growing $\mathrm{ZnO}$ nanoparticles in the matrix of polyethylene glycol, J. Electrochem. Soc. 149, pp. H107-H112 (2002).

4. Abdullah, M., Lenggoro, I. Wuled \& Okuyama, K. \& Shi, Frank G., Insitu synthesis of polymer nanocomposite electrolytes emitting a high luminescence with a tunable wavelength, J. Phys. Chem. B 107, pp. 19571961 (2003).

5. Abdullah, M., Morimoto, T. \& Okuyama, K., Generating blue and red luminescence from $\mathrm{ZnO}$ /polyethylene glycol nanocomposites, prepared by in-situ method, Adv. Func. Mater. 13, pp. 800-804 (2003). 
6. Abdullah, M., Shibamotob, S. \& Okuyama, K., Synthesis of $\mathrm{ZnO} / \mathrm{SiO} 2$ nanocomposite emitting specified luminescence Color, Opt. Mater., in press.

7. Abdullah, M., Panatarani, C., Kim, T.-O. \& Okuyama, K., Nanostructured ZnO/Y2O3:Eu for use as fillers in luminescent polymer electrolyte composites, J. Alloys Comp., in press.

8. Abdullah, M., Lenggoro, I. Wuled \& Okuyama, K., Polymer electrolyte nanocomposites, in Encyclopedia of Nanoscience and Nanotechnology, American Scientific Publisher, in press.

9. Yamamoto, S., Kakihana, M., Kato, S., A polymer complex solution route to the low-temperature synthesis of tetragonal $\mathrm{Zr} 0.88 \mathrm{Ce} 0.12 \mathrm{O} 2$ with a reduced amount of organic substance, J. Alloys Comp. 297, pp. 81-86 (2000)

10. Durán, P., Capel, F., Gutierrez, D., Tartaj, J., Moure, C., Cerium (IV) oxide synthesis and sinterable powders prepared by the polymeric organic complex method, J. Eur. Ceram. Soc. 22, pp. 1711-1721 (2002).

11. Ray, J. C., Pati, R. K., Pramanik, P., Chemical synthesis of nanocrystalline zirconia by a novel polymer matrix-based precursor solution method using triethanolamine, Mater. Lett. 48, pp. 74-80 (2001).

12. Duran, P., Tartaj, J., Moure, C., Sintering behavior of Y2O3 powders prepared by the polymer complex solution method, Ceram. Int. 28, pp. 791-803 (2002).

13. Ito, Y., Abdullah, M. \& Okuyama, K., Direct Preparation for nonagglomerated indium tin oxide nanoparticles using various spray pyrolysis methods, J. Mater. Res., in press.

14. Seinfeld, J. H. \& Pandis, S. N., Atmospheric Chemistry and Physics, NY, Wiley, (1998).

15. Fuchs, N. A., Mechanics of Aerosols, NY, Pergamon, (1964).

16. B. Dahneke, in Theory of Dispersed Multiphase Flow, edited by R.E. Meyer, Acad. Press,NY, pp. 97-138, (1983).

17. H. R. Kyurt, Colloid Science, Amsterdam, Elsevier, (1952). 